\title{
Editorial. Tecnologías digitales, sociedad e Hipertextos en la "nueva normalidad"
}

\author{
Cómo citar: Dirección y Consejo editor Hipertextos (2020). Editorial. Tecnologías digitales, sociedad e \\ Hipertextos en la "nueva normalidad". Revista Hipertextos, 8(14), pp. 7-10. DOI: \\ https://doi.org/10.24215/23143924e017
}

Luego de un año de peculiar intensidad y habiendo modificado nuestra metodología habitual de trabajo, sale a la luz el número 14 de la Revista Hipertextos. Capitalismo, técnica y sociedad en debate.

Esta nueva edición nos encuentra celebrando la incorporación de la Universidad Nacional de Rafaela (UNRaf) a nuestro comité editorial, representada por la Lic. María Belén Romero, coordinadora de Ediciones UNRaf. El área de Comunicación de esa Universidad se encargó de renovar el diseño gráfico de nuestra tapa, que estrenamos en este número 14. Se trata de un nuevo paso en la ampliación del alcance nacional de nuestra Revista, que poco a poco va saliendo del ámbito de la Ciudad de Buenos Aires y enriqueciéndose con miradas y aportes desde diferentes partes del país.

En este sentido es necesario reconocer que la adaptación a la "nueva normalidad" nos trajo algunas ventajas: les colegues que no viven en la ciudad de Buenos Aires -donde, por ahora, vive la mayor parte del comité editorial- pudieron participar de la misma manera que el resto. Antes del aislamiento también lo hacían por medios digitales, generalmente una llamada a través de un celular colocado en el medio de la mesa de la reunión presencial, desde el que emanaban sus voces. Quienes no viven tan lejos, por ejemplo en la ciudad de La Plata, no tuvieron que trasladarse hasta la ciudad de Buenos Aires para nuestros encuentros. Durante 2020 todes pudimos vernos las caras y participar en igualdad de condiciones.

Entre las diferentes adaptaciones que hemos ensayado se encuentra la presentación de la revista, específicamente del número anterior - el 13 - en el Simposio de Tecnología y Sociedad de las $49^{\circ}$ Jornadas de Informática (JAIIO), realizadas de manera virtual. Debemos mucho a la buena voluntad de les autores, que enviaron los videos de presentación de sus artículos y al esfuerzo realizado por la comisión de comunicación de nuestro comité editorial, que incursionó en la edición audiovisual y realizó la difusión del evento.

Estas adaptaciones hubieran resultado imposibles sin acceso a tecnologías digitales e internet o, para decirlo en otros términos, sin estar incluides digitalmente. El año 2020 nos mostró que la deuda en ese sentido continúa, sobretodo cuando tener conexión a Internet y acceso a artefactos que procesan información digital es absolutamente necesario para continuar manteniendo vínculos de toda índole: educativos, laborales, sociales, de acceso a la salud, inclusive para gestionar la movilidad espacial que podemos tener conforme suben o bajan los casos de COVID19.

En este sentido, celebramos la declaración de los servicios de Internet, la telefonía celular y fija y la televisión paga como servicios públicos esenciales, a partir del Decreto 609/20 del Poder Ejecutivo Nacional. Esto implica que el Estado es responsable de asegurar el acceso universal a este tipo de servicios dentro de marcos normativos que prevean la rendición de cuentas para las empresas que los provean, sean públicas o privadas. También abre la posibilidad a la existencia de planes inclusivos de prestación básica, universal y obligatoria para las personas de menores 
recursos. Asimismo, se ordenó congelar las tarifas de Internet, telefonía celular y televisión paga hasta el 31 de diciembre de 2020.

$\mathrm{Si}$ bien desde el gobierno nacional se ha procurado generar acuerdos con empresas de telefonía móvil para evitar el consumo de datos en sitios con dominio .edu.ar, gran parte de la actividad educativa, tratando de emular lo presencial, tuvo lugar a través de plataformas privadas de videoconferencias, las que no siempre estaban integradas a los sitios .edu.ar institucionales.

Al mismo tiempo, la situación de aislamiento impuesta por la pandemia generó un gran mercado para los sistemas de videoconferencias, especialmente Zoom, Google Meet y Webex que, si bien proveen gratuitamente un tiempo limitado de conexión, son empresas con fines de lucro y con graves problemas vinculados a la privacidad de sus usuarios. En ese sentido, entendemos que, puesto que los datos de los usuarios quedan en manos de las empresas que proveen esos servicios y obtienen ganancias exorbitantes, en un mediano plazo es importante promover herramientas públicas, estatales o no estatales, para tales usos. En el corto plazo, la utilización obligatoria de este tipo de aplicaciones debería limitarse a actividades que por sus características requieran necesariamente de la interacción en tiempo real, con el objetivo de no excluir a quienes no cuentan con los artefactos y/o conexiones adecuadas. Esto no quiere decir que sea necesario aceptar esa situación sino que, por el contrario, se trata de una deuda urgente y primordial que saldar.

No solo los sistemas de videoconferencias han crecido exponencialmente, también lo han hecho todas las plataformas digitales dedicadas a la comercialización de diversos productos y servicios, incluidas en lo que ha comenzado a denominarse capitalismo de plataformas. Cuatro de las empresas de tecnología más grandes de EE. UU.: Amazon.com Inc., Apple Inc., Facebook Inc. y Alphabet Inc. crecieron exponencialmente durante 2020 haciendo subir los precios de sus acciones. Las de Amazon, por ejemplo, cotizan aproximadamente un 30\% más alto que el promedio de los últimos cinco años (Kim, 2020).

En este número de la Revista Hipertextos. Capitalismo, técnica y sociedad en debate, se abordan estos y otros temas que cobran especial relevancia en el contexto de digitalización de nuestra vida cotidiana, signada por diversas formas de interacción digital impuestas por el aislamiento y/o distanciamiento a causa de la pandemia de COVID19.

\section{Artículos}

En primer lugar, como es habitual, Hipertextos pone a disposición de los lectores de habla hispana la traducción de un texto originalmente escrito en inglés, que por su temática es afín a los intereses de los lectores de la Revista. Se trata de un texto de Florian Schmidt, titulado "Mercados de trabajo digitales en la economía de plataformas. Mapeando los desafíos políticos del trabajo colaborativo y del trabajo de plataformas". La traducción fue realizada por Agostina Dolcemáscolo, Bernadette Califano, Carolina Monti, Emilio Cafassi, Guillermo Quiña y Martina Lassalle, bajo la coordinación de Esteban Magnani, quien también realizó la revisión y corrección de la versión final; todes integrantes del Comité Editorial de Hipertextos.

El artículo analiza los modelos de negocios basados en el uso de plataformas digitales y sus implicancias para la economía y la sociedad. Se centra en las que se dedican al mercado de trabajo digital, entre ellas Uber, Airbnb, Helpling. Explica los mecanismos de estos mercados y propone puntos de partida para la toma de decisiones políticas. Estas nuevas plataformas median entre la oferta y la demanda y, si bien son una fuerza impulsora de la innovación económica, dependen 
de una fuerza laboral conformada por personas que trabajan por su propia cuenta y riesgo, por salarios bajos y sin ningún tipo de seguridad social.

Continuando con una perspectiva centrada en la economía y atravesada por lo digital, el artículo "La economía de la atención: del ciber-tiempo al tiempo cinemático", de Claudio Celis Bueno, parte de la noción de tiempo cinemático de Bernard Stiegler para examinar cómo el tiempo humano y el ciber-tiempo se constituyen mutuamente, contrariamente a lo que postula la economía de la atención, concepto nacido en el campo de la economía política y que naturaliza una noción de tiempo humano como privado de cualquier tipo de transformación histórica, razón por la cual la diferencia entre las posibilidades humanas para prestar atención y procesar información y la velocidad a la que esta fluye, resulta imposible de saldar.

Pasando a las temáticas relacionadas con el poder en un mundo atravesado por las tecnologías digitales, el artículo "Las teorías postdisciplinarias y el desafío de describir una nueva tecnología del poder", de Jonathan Enrique Prueger, aporta a la caracterización de una nueva tecnología de poder (psicopolítica/noopolítica) partiendo desde teorías foucaultianas y postfoucaultianas, intentando reactualizar sus herramientas analíticas en un contexto donde la pandemia del COVID-19 vuelve a dar protagonismo a los dispositivos y técnicas de la biopolítica. Al mismo tiempo, aborda a autores como Lazzarato y Han para integrar los registros del ciberespacio, los signos, la memoria, con aquellos que atañen a una perspectiva centrada en la psique, el inconsciente y las políticas del deseo.

El artículo "Tecnologías de vigilancia en las empresas mexicanas para protegerse de la inseguridad”, de Lucía Carmina Jasso López, estudia la aplicación de medidas preventivas que consideran a las tecnologías de vigilancia -alarmas y cámaras de video- como una alternativa para protegerse de la inseguridad. Se trata de un trabajo de investigación que parte del análisis estadístico de encuestas de victimización de empresas en México para analizar el incremento de la inversión en tecnologías de vigilancia.

En relación con la información científica en formato digital, el artículo "Acceso a la literatura científica desde Sci-Hub: Análisis y reflexión de las descargas en Argentina”, escrito por Carolina Monti y Carolina Unzurrunzaga, analiza el uso que se hace de Sci-Hub en Argentina a partir de un análisis cuantitativo de las estadísticas de descargas disponibles hasta 2017, en un contexto donde la comercialización de la información científica y las barreras de pago para su acceso son problemáticas muy debatidas en las últimas décadas. Sci-Hub es un repositorio web nacido en Rusia y fundado por Alexandra Asanovna Elbakyan en 2011. Cuenta con más de 70 millones de artículos académicos a los que puede accederse sin pago previo ni entrega de datos personales. En el presente artículo se detectan patrones de uso generales, editoriales y temáticos de los documentos y se indaga en aspectos comunes y diferenciadores con respecto al uso mundial y en otros países de América Latina. También se determina si los artículos accedidos están además disponibles en acceso abierto o si se puede acceder a ellos a través de las suscripciones pagadas por la Biblioteca Electrónica de Ciencia y Tecnología (BECyT). Además, se postula que mientras la información científica siga siendo una mercancía es primordial profundizar el estudio de los distintos proyectos que permiten la reapropiación del conocimiento.

Finalmente, y continuando con la temática de acceso al conocimiento, el artículo "Conocimientos doblemente libres en la expansión sistémica de la propiedad intelectual" de Antonela Isoglio busca, a partir de un estudio de caso que incluye como unidades de análisis los proyectos GNU, Perl y GenBank, comprender los flujos de conocimiento que adoptaron el carácter de conocimientos doblemente libres, es decir, aquellos que se caracterizan por circular 
con restricciones mínimas de uso y que, al mismo tiempo, su utilización no genera remuneración para sus productores.

\section{Reseña}

En esta sección, inaugurada ya algunos números atrás, Esteban Magnani realiza una reseña de The age of surveillance capitalism (Hachette Book Group, 2019) de Shoshana Zuboff, aún no traducido al castellano. Esta obra indaga la sociedad actual a partir de mecanismos ocultos tras algoritmos para la acumulación de dinero, datos y conocimiento técnico. Analiza el modelo de negocios de diversas empresas basadas en tecnologías digitales y lo asimila a una nueva forma de acumulación a la que denomina capitalismo de vigilancia. Si bien en todos los modos de producción se generó alguna forma de sujeción, en la actualidad esta capacidad se magnifica, incluyendo al trabajo, la familia, las relaciones sociales y, también, a la vida interior de los sujetos como espacios de reproducción del capital. Esto se logra a través de la transformación de nuestras vidas en datos que son reconfigurados en nuevas formas de control social. No se trata de una capacidad meramente tecnológica, sino que es parte también de un proyecto político neoliberal que busca manejar las emociones de las personas.

\section{Debate}

La sección de Debate es una invitación a la reflexión en torno a las prácticas educativas llevadas a cabo para sostener la continuidad de las cursadas en el ámbito de la educación superior. El artículo "La educación remota de emergencia y los peligros de imitar lo presencial", de Ana Marotias, pone en discusión una serie de supuestos y prejuicios en torno de la educación a distancia digital e intenta dilucidar las diferencias entre la educación remota de emergencia, surgida para garantizar el derecho a la educación en el contexto del aislamiento social, preventivo y obligatorio y la clásica educación a distancia. Al mismo tiempo insiste en dejar claro que es imposible -y poco deseable- tratar de imitar a la educación presencial, sub-utilizando de esta manera las potencialidades de lo virtual y generando situaciones de exclusión. Finalmente, propone algunas alternativas a esta disyuntiva de cara a una transformación de la educación superior que continuará luego de superada la pandemia, Sputnik V, Oxford-AstraZeneca, Sinopharm, Pfizer y demás vacunas mediante.

La dirección y el consejo editor

Diciembre de 2020 